\title{
Influences of geo-spatial location on pre-exposure prophylaxis use in South Africa: positioning microbicides for better product uptake
}

\section{Eliza M. Govender, Leila E. Mansoor \& Quarraisha Abdool Karim}

To cite this article: Eliza M. Govender, Leila E. Mansoor \& Quarraisha Abdool Karim (2017) Influences of geo-spatial location on pre-exposure prophylaxis use in South Africa: positioning microbicides for better product uptake, AIDS Care, 29:6, 734-740, DOI: 10.1080/09540121.2016.1248349

To link to this article: http://dx.doi.org/10.1080/09540121.2016.1248349

曲 Published online: 01 Nov 2016.

Submit your article to this journal ๔

Џ Article views: 77

Q View related articles $匚$

View Crossmark data $[\pi$ 


\title{
Influences of geo-spatial location on pre-exposure prophylaxis use in South Africa: positioning microbicides for better product uptake
}

\author{
Eliza M. Govender ${ }^{\mathrm{a}, \mathrm{b}}$, Leila E. Mansoor ${ }^{\mathrm{a}}$ and Quarraisha Abdool Karim ${ }^{\mathrm{a}, \mathrm{c}}$ \\ ${ }^{\mathrm{a} C}$ Centre for the AIDS Programme of Research in South Africa (CAPRISA), University of KwaZulu-Natal, Durban, South Africa; ${ }^{b}$ Department of \\ Culture, Communication and Media Studies, School of Applied Human Sciences, University of KwaZulu-Natal, Durban, South Africa; \\ 'Department of Epidemiology, Mailman School of Public Health, Columbia University, New York, NY, USA
}

\section{ABSTRACT}

Young women bear a disproportionately high burden of HIV infection in sub-Saharan Africa, prioritising pre-exposure prophylaxis (PrEP) can be an integral part of HIV prevention combination strategies. Women initiated HIV prevention technology options will require consistent adherence, an imperative for product effectiveness. With several PrEP clinical trials underway; exploring women's acceptability to advances in HIV prevention technologies can better facilitate demand creation for future PrEP roll out. This study utilised the opportunity of post-trial access to CAPRISA 008 women (trial) and non-trial women from three geo-spatial settings (urban, rural and peri-urban) to identify microbicide acceptability and how product associations of microbicides can influence future HIV prevention choices. Six participatory workshops using participatory action research with art-based activities and discussion groups were conducted in KwaZulu-Natal with 104 women from various geo-spatial locations and social status to understand microbicide acceptability and product associations. The data were analysed using thematic analysis. The study found that women's acceptability and product association of the tenofovir gel microbicide differed according to rural and urban areas. Most urban women identified confidence, sexiness and classiness as key associations that will encourage microbicide acceptability and use, while rural women identified respect, responsibility and confidence as the key product associations, with increased focus on the individual and collective family/community benefits of product acceptance and use. Urban-rural differences suggest a market segmentation that is contextualised to be locally responsive to promote HIV prevention technologies. Various sexual encounters further determined the types of HIV prevention technologies women would consider. In line with WHO's recommendation that PrEP should be an additional prevention choice for people at risk of HIV, this study underscores the importance of user engagement, understanding product associations and how this can influence product acceptability and promotion of HIV prevention technologies.
ARTICLE HISTORY

Received 20 June 2016

Accepted 10 October 2016

\section{KEYWORDS}

Pre-exposure prophylaxis; participatory research; market segmentation

\section{Introduction}

HIV incidence rates have slowed down globally, yet subSaharan Africa still has one of the highest rates of new HIV infections (UNAIDS, 2015). Although the epidemic affects both men and women, $60 \%$ of new infections in South Africa occurs among young women (Shisana et al., 2014). South Africa has one of the highest numbers of HIV infections, (UNAIDS, 2014) with the highest HIV prevalence rates in KwaZulu-Natal. Young women (aged 15-24 years) bear a disproportionate burden of HIV infection, having up to eight times more infection compared to their male peers (Dellar, Dlamini, \& Abdool Karim, 2015), and acquire HIV infection at least five to seven years earlier than men (UNAIDS, 2014). In the age group 15-49 years, prevalence of HIV amongst female was 23, 3\% and 13,3\% among South African males (Shisana et al., 2014), indicating that women need more sustainable HIV prevention options. With limited safe and effective women-centred HIV prevention options, progress on pre-exposure prophylaxis (PrEP) has the potential to reduce transmission of HIV.

Since 2010, the outcomes of several trials have demonstrated the prevention benefit of the prophylactic use of antiretrovirals that has generated new hope in preventing sexually acquired HIV infection. Several studies of daily and intermittent dosing strategies in different settings, populations and modes of transmission have confirmed the prevention benefits of tenofovir-based products in HIV-uninfected persons (Abdool Karim et al., 2010; Baeten et al., 2012; Cohen et al., 2011; Grant et al., 2010; McCormack et al., 2016; Molina et al., 2015; Thigpen et al., 2012). For the first time, the

CONTACT Eliza M. Govender eliza.govender@caprisa.org Centre for the AIDS Programme of Research in South Africa (CAPRISA), University of KwaZuluNatal, 2nd Floor, Doris Duke Medical Research Institute, 719 Umbilo Road, Durban 4041, South Africa 
most recent WHO ARV Guidelines released in September 2015 makes a strong recommendation for the provision of tenofovir-containing PrEP to be offered as an additional prevention choice for people at substantial risk of HIV infection as part of combination HIV prevention approaches (WHO, 2015).

An expanding HIV PrEP pipeline with several innovations under clinical trial will result in women having a buffet of HIV preventative options. As product development, formulations and dosing strategies/options expand, and as evidence of efficacious prevention products emerge, there is an urgent need for an in-depth understanding of what influences product acceptability. Studies focusing on microbicides in the form of vaginal gels and rings, which have already undergone clinical trials, can provide critical information about potential users' product perceptions which can influence microbicide acceptability. Microbicides are likely to be part of a comprehensive HIV prevention strategy in the future, existing research on microbicides suggest that there is a need to investigate factors that may contribute to the acceptance, uptake and adherence of microbicides as HIV prevention technologies (Kelly et al., 2015; Kippax, 2012; MacQueen et al., 2014), with a specific focus on the acceptability of microbicides in a cross-cultural context (Greene et al., 2010; Mantell et al., 2005). This study utilised the opportunity to work with CAPRISA 008 post-trial participants (rural and urban) and extended to women who have not participated in clinical trials (rural, peri-urban and urban) to explore their acceptability of microbicides and specific product associations that can influence the future uptake of HIV prevention technologies. The mix of trial and non-trial women was to ascertain product associations from women who used the gel previously and those who were introduced to the gel for the first time.

\section{Methods}

\section{The intervention}

This study used participatory action research (PAR) as a process of engaging women in ongoing dialogue to understand often complex, multi-dimensional, intractable and dynamic problems (Brydon-Miller, 2003; Brydon-Miller, Greenwood, \& Maguire, 2003, p. 21), such as acceptability of microbicide use. The PAR process engaged women in a collective and cyclical process of discussion within the context of female-initiated HIV prevention options. PAR provides a way for women to take part in "the process of generating knowledge and advocating positive social change in order to promote more effective health care practices" (Brydon-Miller, 2003, p. 187). The women attending the workshops were provided with large sheets of paper, and through a facilitated process, asked to write, draw or discuss their ideas about their own associations with potential microbicide use. A brief background to HIV prevention, the need for more comprehensive HIV prevention options for women, the advancement of clinical trials and the introduction to vaginal microbicides and dosing strategies informed the introduction to microbicides using visual aids. The workshops were conducted in English with isiZulu translations where necessary for clarification. The women were provided with a range of oil pastels, markers and coloured paper and given 60 minutes to work in groups of 3 or 4 women to collectively document and discuss their product association with potential microbicide use. The documented artwork was not analysed but used as a catalyst to initiate a knowledge sharing process through group discussions. After smaller group engagement and reflection, bigger discussion groups were convened. These were facilitated by the first author with assistance from a bi-lingual facilitator. In line with PAR techniques, journal notes were taken of observations, with auto recording of discussions. The discussions were transcribed and the text analysed using thematic analysis to establish the key product associations that can influence microbicide acceptability.

\section{Sampling}

Convenience sampling was used to recruit women to attend these workshops, with the key eligibility criteria that all women had to be over 18 years of age. Whilst HIV negative status is a determinant for future eligibility for microbicide use, knowledge of HIV status was not a requirement for sampling. DramAidE (Drama in AIDS Education), a non-governmental organisation facilitated the recruitment of non-trial women in eThekwini (urban) and Umnini (peri-urban), with COMOSAT (Community Outreach Programme in Vulindela) assisting with recruitment of non-trial women in Vulindela (rural). Women who participated in the tenofovir gel clinical trial were asked to volunteer to have their contact details shared with the researcher, and if they agreed their permission was documented in their trial records. A random selection of trial participants were contacted for participation in this study. The inclusion of trial women contributed to the discussion of product associations from women who were exposed to the gel use and those who were introduced to the gel for the first time. Trial women were specifically included in the study not for comparative analysis but as good PAR practice (McIntyre, 2009), to ensure that they were not only part of the clinical trial phase but also contributed to understanding microbicide acceptability. The researcher anticipated that through the inclusion of trial participants without a direct 
disclosure of their involvement, it will provide a space for inclusion of their perspectives of microbicide acceptability and product associations. Informed consent was obtained from all trial and non-trial women. Ethical clearance was obtained from the Human and Social Science Ethics Committee of the University of KwaZulu-Natal, South Africa.

\section{Workshop composition}

Women were recruited from three geo-spatial locations (urban, peri-urban and rural) for a diverse representation of potential microbicide users. Trial and nontrial women were included in this study, with trial women being those who had used the tenofovir gel in a clinical trial and non-trial women are those who have not used the gel or enrolled into any clinical trials underway. Participants were briefed that the product is a clinical trial product, and can reduce HIV infection by up to $39 \%$ if adhered to consistently (Abdool Karim et al., 2010). They were also briefed on the benefits of gel use, the application process and the need to still consider other HIV preventative measures. All participants were informed that the product was not available at the time of the study, but the purpose is to get an in-depth understanding of their acceptability.

A total of 6 workshops were conducted with convenience sampling of 20 women for each of the workshops. Not all of the women recruited attended; hence each workshop had a varying number of women in attendance (see Table 1). The eThekwini mixed group had increased numbers due to participants self-inviting other women interested in the study, given the PAR methodology of the study, these additional women were not denied participation in the study. Two of the six workshops were conducted with mixed groups of trial and non-trial women in urban (eThekwini) and rural (Vulindela) settings, as these were the sites for the CAPRISA008 implementation study. Mixed groups of trial and nontrial women from the various geo-spatial locations were used to ensure that the confidentiality of the trial women was still maintained when working with the facilitators: facilitators were unaware of who were the trial and non-trial women. Four of the six workshops were conducted with non-trial women only in a rural (Vulindela), peri-urban (Umnini) and two urban sites (eThekwini).

The two urban sites purposively selected two varying groups of women; a student group between the ages of 18-24 years, and a working professionals group between ages of 25-49 years to understand the microbicide acceptability and product associations of women from various ages and social status. Women selected from urban, rural and peri-urban locations and from varying age groups and social status catered for a more nuanced understanding of microbicide acceptability and its influence on future product uptake.

The data presented below are the key themes from the data analysed highlighting the influence geo-spatial locations and varying types of sexual encounters on microbicide acceptability. Thematic analysis was conducted where repetition of common issues were identified as the central themes for discussion. The broader themes were refined into specific codes with two coders reading the text to identify common themes and codes. The codes were compared periodically to ensure that the codes were similar.

Overall the study was limited in understanding the actual trial participant acceptability of microbicides as the focus groups were not constituted to offer this comparative data of clinical trial users and potential users, in addition further research that extends to more sites of urban and rural locations can enhance understanding on potential microbicide acceptability.

\section{Results}

\section{Confident women use microbicides}

Microbicide use was accepted as a female-initiated prevention option with many associating that only

Table 1. Breakdown of workshop sample.

\begin{tabular}{|c|c|c|c|c|}
\hline Geo-spatial location & Trial/non-trial women & Age & Social status (occupation) & Total in attendance \\
\hline Vulindela (rural) & $\begin{array}{l}\text { Mixed (trial and non- } \\
\text { trial women) }\end{array}$ & $\begin{array}{l}18-55 \\
\text { years }\end{array}$ & $\begin{array}{l}\text { Stay at home moms, domestic workers and un- } \\
\text { employed }\end{array}$ & 14 \\
\hline eThekwini (urban) & $\begin{array}{l}\text { Mixed (trial and non- } \\
\text { trial women) }\end{array}$ & $\begin{array}{l}18-55 \\
\text { years }\end{array}$ & $\begin{array}{l}\text { Stay at home moms, domestic workers/cleaners } \\
\text { and admin jobs/un-employed }\end{array}$ & 31 \\
\hline Umnini (peri-urban) & Non-trial women & $\begin{array}{l}18-55 \\
\text { years }\end{array}$ & $\begin{array}{l}\text { Stay at home moms, domestics, part time } \\
\text { students and un-employed }\end{array}$ & 16 \\
\hline Vulindela (rural) & Non-trial women & $\begin{array}{l}18-55 \\
\text { years }\end{array}$ & $\begin{array}{l}\text { Stay at home moms, domestic workers and un- } \\
\text { employed }\end{array}$ & 10 \\
\hline eThekwini (urban students) & Non-trial women & $\begin{array}{l}18-24 \\
\text { years }\end{array}$ & $\begin{array}{l}\text { Students studying full or part time at private } \\
\text { colleges or universities }\end{array}$ & 16 \\
\hline \multirow[t]{2}{*}{$\begin{array}{l}\text { eThekwini (urban working } \\
\text { professionals) }\end{array}$} & Non-trial women & $\begin{array}{l}25-55 \\
\text { years }\end{array}$ & Professional working women & 17 \\
\hline & & & & $\begin{array}{l}104 \text { women (45 mixed group women } \\
\text { and } 59 \text { non-trial women) }\end{array}$ \\
\hline
\end{tabular}


"confident women" would use the product. A "confident woman" was described as someone who understands the importance of protecting herself, and is able to take the relevant steps to ensure she adheres to the product.

The terms "confident" were commonly associated with women who accept microbicide use, however women in the urban and rural areas ascribed subtly different meanings to the term confident. The urban women's notion of a confident woman was associated with "sexiness": the use of vaginal microbicides suggested a feeling of sexiness and confidence by lowering the risk of HIV infection. Urban women directly related potential microbicide use to women feel sexier and hence more confident about sex, where confidence was used to describe microbicide use as empowering and taking responsibility for an individual's sexual well-being. Sexiness was defined for urban women as feeling more physically appealing when having the freedom and confidence to prevent HIV infection.

The rural women on the other hand, associated microbicide use with "confidence" and "respect" where "respect" signified the individual and collective responsibility of reducing the risk of HIV infection. The acceptability of microbicides in rural areas suggested that women were collectively taking steps to protect themselves, making them confident about their responsible sexual choices. The rural women's confidence was associated with an individual agency of protection, yet in considering microbicide use rural women are respected for protecting themselves and their family, extending microbicide acceptability to a wider collective community benefit. The use of the term "confidence" was strongly advocated for both mixed groups in urban and rural settings, suggesting that some women could have responded from their position of actual product use.

\section{Location determines microbicide use}

Women from all locations believed that the gel was an opportunity to protect themselves in the absence of condom negotiation, increase of sexual assault and domestic violence, partner unfaithfulness and male dominance. Women in rural areas were more likely to use microbicides as many knew when they will see their partners, or when they are returning home from long periods of work - gel application was identified as an easy process in these instances. Urban student's acceptance and potential for product uptake differed depending on the campus residential and non-residential living arrangements; those who lived at home with parents identified the need to secretly store the gel as a deterrent of product use. Those living at campus/university residence had more freedom with HIV prevention product choices. The working urban women identified that many who were in long-term relationships may not require the gel use, unless they suspected partners unfaithful. The peri-urban women's uptake of microbicides were linked to the long distance many young girls had to walk to school, and their increased exposed to risk through opportunities for transactional sex and cases of rape.

Overall the rural women's acceptability of microbicides was associated with the women's ability to feel proud of her step towards reducing risk of HIV infection. While many identified the importance of the product empowering women to prevent HIV in cases where men were unwilling to use the condom, rural women had a greater acceptability for microbicide use when men were supportive of their HIV prevention choices. For most rural women, acceptability of microbicides was empowering for women but male support of female-initiated prevention reaffirmed the importance of trust, openness and the willingness to talk about microbicide use to their family and friends.

This was contradictory for urban women where microbicides had increased acceptability due to covert use. Urban women identified an increased receptivity to microbicide use due to the discreet application, whereas HIV prevention product visibility (i.e., female condoms) would pose issues of trust and assumptions of unfaithfulness if used in long-term relationships. While both groups of rural and urban women identified that microbicide acceptability was linked to women feeling empowered and having agency to take individual responsibility, the urban-rural contrast of understanding this agency suggested that male support of femaleinitiated prevention would increase microbicide acceptability in rural locations, while urban women were in favour of product privacy and secrecy of microbicide use.

\section{Varying sexual encounters influence microbicide acceptability}

The rural and peri-urban women were less prescriptive in who the specific target group of women should be for microbicide use in the future. Instead they strongly advocated that microbicides should be used by all sexually active women.

The urban women identified that microbicides in the form of gel was not for all sexually active women. The urban lifestyle was described as being very different to rural settings: seeing partners more regularly, living with their partner and having a more spontaneous social life meant that gel use varied according to urban lifestyles. The working class women in particular identified that living with or seeing their partners spontaneously 
made the dosing strategy less appealing as sex is not always a rational activity. However, the gel could work for women in stable relationships but were aware of their partner/husband's closet (secretive) relationships and needed a discreet way to still be able to protect themselves. The urban students compared the varying lifestyles of living with parents, living alone and studying away from home as a key variable in microbicide acceptability and future uptake. Many discussed the complexities of storing the gel if residing with parents, as opposed to living in university residence. Students found that the gel's dosing strategy was less appealing for them, giving the often-impromptu nature of their sexual engagement. Many suggested that microbicide uptake must consider these types of varying sexual relationships and ever changing sexual landscapes. Overall, urban women lifestyles and residential variances influenced the acceptability of gel microbicide use.

Tenofovir gel acceptability had considerable merit in cases where women were knowledgeable about when they were likely to have sex. Many urban women suggested that the gel would work well in cases of planned and scheduled sexual encounters as long as the product was consistently adhered too. Transactional sex, women in relationships with migrant workers, "sugar daddy" relationships and cases of where sex is a planned encounter, would be favourable opportunities for the consistent and correct application of the gel. Most urban women (students and working professionals only) argued that the BAT24 dosing strategy (up to 12 hours before sex, and up to 12 hours after sex) created several product limitations, as most sexual encounters were unplanned and spontaneous. The routine application of the gel negatively influenced consistent adherence. Women from peri-urban and rural locations on the other hand, had a better indication of when they would see their partners or when they were likely to return home from jobs in the city. The gel, if adhered to consistently, could work well in cases of planned and rational sexual encounters. Given the BAT24 dosing strategy, women who engage in scheduled or routine sex would mostly benefit from the product.

\section{Discussion}

Understanding who are the potential microbicide users and the receptivity of female-initiated HIV prevention offers critical information to advance PrEP product development and implementation. The data above highlight a distinct urban-rural disparity on microbicide acceptability for reducing the risk of HIV infection. The data collected in KwaZulu-Natal across rural, periurban and urban settings clearly suggest that the dosing strategy works only for specific planned sexual encounters and hence creates a market segmentation among women in terms of HIV preventative measures. Geospatial contexts are important as women's preventative preferences can be influenced by the type of sexual relationships and where they reside, and underscores the importance of participatory research to inform and match new biological products with the right cohort of potential women and users. Future HIV prevention technologies will need to prioritise an understanding of the potential users, their patterns of various sexual behaviours in various locations and the predictors of product acceptability to encourage instant adoption of femaleinitiated prevention once available (see McMahon, Myers \& Kurth, 2014; Young \& McDaid, 2014).

Extrapolating from this research, we can apply the results of this study to think about how to promote product acceptability for PrEP products still undergoing clinical trial. The research highlights the importance of conducting ongoing research to understand the match between what will influence acceptability of HIV prevention technologies and how best to position PrEP for future demand. Studies conducted on microbicide use in Kenya further highlighted the importance of tailored microbicide communication materials for women who do not use HIV prevention methods and those who have a high-risk perception (Ryan et al., 2015; Sidibe et al., 2014). The HIV pandemic from inception has demonstrated the complexities of human behaviour, and the multiple biological, social, economic, cultural and environmental factors that need to be considered when trying to promote behaviour change (Dutta, 2008, 2011, 2013). These biomedical options for women are a first step and opportunity to customise HIV risk reduction strategy with specific sexual behaviours and practices. While PrEP options such as the vaginal microbicide can promote HIV prevention and be a tool for empowerment of women, a context-specific approach is necessary to understand how women define confidence, respect, responsibility and empowerment.

With several clinical trials underway, women may for the first time in three decades have a variety of options to select the most context-specific HIV prevention technologies, which can be available in a variety of formulations and delivery mechanisms (Celum et al., 2015). Notwithstanding that they are in different stages of development they enable a different approach to introduction and evaluation of each option: this allows better matching of product with what women need in different epidemic settings linked to perception of risk; nature and timing of coital encounters; and other needs, desires and preferences that women have or need from HIV prevention technologies. 


\section{Conclusion}

The findings of this study overall suggest that acceptability of microbicides are significantly influenced by understanding the localised and cultural context in which women make sense of female-initiated HIV prevention methods. Understanding the product associations of the women according to geo-spatial locations and types of sexual encounters can better position PrEP for future demand creation.

\section{Acknowledgements}

We pay tribute to the women who participated in this research; their dedication and commitment made this study possible. We also acknowledge and thank Dr Kathleen (Kate) MacQueen for her comments and input in the drafting of this manuscript.

\section{Disclosure statement}

No potential conflict of interest was reported by the authors.

\section{Funding}

This work was supported by the Centre for the AIDS Programme of Research in South Africa (CAPRISA) and the MACAIDS Fund through the Tides Foundation (Grant \# TFR11-01545).

\section{References}

Abdool Karim, Q., Abdool Karim, S. S., Frohlich, J. A., Grobler, A. C., Baxter, C., Mansoor, L. E., ... Taylor, D. (2010). Effectiveness and safety of tenofovir gel, an antiretroviral microbicide, for the prevention of HIV infection in women. Science, 329(5996), 1168-1174.

Baeten, J. M., Donnell, D., Ndase, P., Mugo, N. R., Campbell, J. D., Wangisi, J., ... Celum, C. (2012). Antiretroviral prophylaxis for HIV prevention in heterosexual men and women. The New England Journal of Medicine, 367(5), 399-410.

Brydon-Miller, M. (2003). Using action research methodologies to address community health issues. In M. Murray (Ed.), Critical health psychology (pp. 217-233). London: Palgrave.

Brydon-Miller, M., Greenwood, D., \& Maguire, P. (2003). Why action research? Action research (Vol. 1). London: Sage.

Celum, C. L., Delany-Moretlwe, S., McConnell, M., van Rooyen, H., Bekker, L.-G., Kurth, A. ... Beaten, J. M. (2015). Rethinking HIV prevention to prepare for oral PrEP implementation for young African women. Journal of the International AIDS Society, 18(Suppl. 3), 20227. doi:10.7448/IAS.18.4.20227

Cohen, M. S., Chen, Y. Q., McCauley, M., Gamble, T., Hosseinipour, M. C., Kumarasamy, N., ... Fleming, T. R. (2011). Prevention of HIV-1 infection with early antiretroviral therapy. The New England Journal of Medicine, 365, 493-505.
Dellar, R. C., Dlamini, S., \& Abdool Karim, Q. A. (2015). Adolescent girls and young women: Key populations for HIV epidemic control. Journal of International AIDS Society, 18(2 Suppl. 1), 64-70.

Dutta, M. J. (2008). Communicating health: A culture-centered approach. Cambridge: Polity Press.

Dutta, M. J. (2011). Communicating social change: Structure, culture and agency. New York, NY: Routledge.

Dutta, M. J. (2013). Disseminating HIV pre-exposure prophylaxis information in underseved communities. American Journal of Preventive Medicine, 44(1 Suppl. 2), S133-S136.

Grant, R. M., Lama, J. R., Anderson, P. L., McMahan, V., Liu, A. Y., Vargas, L., ... Glidden, D. V. (2010). Preexposure chemoprophylaxis for HIV prevention in men who have sex with men. The New England Journal of Medicine, 363(27), 2587-2599.

Greene, E., Btona, G., Hallad, J., Johnson, S., Neema, S., \& Tolley, E. E. (2010). Acceptability and adherence of a candidate microbicide gel among high-risk women in Africa and India. Culture, Helath and Sexuality: An International Journal for Research, Intervention and Care, 12(7), 739-754.

Kelly, C. A., Friedland, B. A., Morar, N. S., Katzen, L. L., Ramjee, G., Mogkatle, M. M., \& Ahmed, K. (2015). To tell or not to tell: Male partner engagement in a phase 3 microbicide efficacy trial in South Africa. Culture, Health \& Sexuality: An International Journal for Research, Intervention and Care, 7(8), 1004-1020.

Kippax, S. (2012). Effective HIV prevention: The indispensable role of social science. Journal of the International AIDS Society, 15(2), 1-8.

MacQueen, K. M., Tolley, E. E., Owen, D. H., Amico, K. R., Morrow, K. M., Moench, T. R., ... Friedland, B. (2014). An interdisciplinary framework for measuring and supporting adherence in HIV prevention trials of ARV-based vaginal rings. Journal of the International AIDS Society, 17(3), 19158. doi:10.7448/IAS.17.3.19158

Mantell, J. E., Myer, L., Carballo-Dieguez, A., Stein, Z., Ramjee, G., Morar, N. S., \& Harrison, P. F. (2005). Microbicide acceptability research: Current approaches and future directions. Social Science and Medicine, 60, 319-330.

McCormack, S., Dunn, D. T., Desai, M., Dolling, D. I., Gafos, M., Gilson, R., ... Gill, O. N. (2016). Pre-exposure prophylaxis to prevent the acquisition of HIV-1 infection (PROUD): effectiveness results from the pilot phase of a pragmatic open-label randomised trial. Lancet, 387 (10013), 53-60.

McIntyre, A. (2009). Participatory action research: Qualitative research methods. Series 52. London: Sage.

McMahon, J. M., Meyers, J. E., Kurth, A. E., Cohen, S. E., Mannheimer, S. B., Simmons, J., ... Haberer, J. E. (2014). Oral Pre-exposure prophylaxis (PrEP) for prevention of HIV in serodiscordant heterosexual couples in the United States: Opportunities and challenges. AIDS Patient Care and STDs, 28(9), 462-474.

Molina, J. M., Capitant, C., Spire, B., Pialoux, G., Chidiac, C., Charreau, I., ... Delfraissy, J.-F. (2015, February 23-26 ). On Demand PrEP with oral TDF-FTC in MSM: Results of the ANRS Ipergay trial. [Abstract 23LB]. Conference on Retroviruses and Opportunistic Infections (CROI). Seattle, Washington.

Ryan, E., Bokh, E., Tolley, E. E., Pack, A. P., Mackenzie, C., Olawo, A., \& Githuka, G. (2015). Positioning microbicides 
for HIV prevention in Kenya: A case study. Social Marketing Quarterly, 21(2), 100-114.

Shisana, O., Rehle, T., Simbayi, L. C., Zuma, K., Jooste, S., Zungu, N.,... Onoya, D. (2014). South African national HIV prevalence, incidence and behaviour survey, 2012. Cape Town: HSRC Press.

Sidibe, S., Pack, A. P., Tolley, E. E., Ryan, E., Mackenzie, C., Bockh, E., \& Githuka, G. (2014). Communicating about microbicides with women in mind: Tailoring messages for specific audiences. Journal of the International AIDS Society, 17(Suppl. 2), 19151. doi:10.7448/IAS.17.3.19151

Thigpen, M. C., Kebaabetswe, P. M., Paxton, L. A., Smith, D. K., Rose, C. E., Segolodi, T. M., ... Brooks, J. T. (2012). Antiretroviral preexposure prophylaxis for heterosexual HIV transmission in Botswana. The New England Journal of Medicine, 367(5), 423-434.
UNAIDS. (2014). The Gap report. 2014. Retrieved April 22, 2015, from http://www.unaids.org/en/resources/document s/2014/20140716_UNAIDS_gap_report

UNAIDS. (2015). Biomedical AIDS research: Recent and upcoming advances. Retrieved September 22, 2015, from http://www.unaids.org/sites/default/files/media_asset/JC27 23_Biomedical_AIDS_research_en.pdf

World Health Organization. (2015). Guideline on when to start antiretroviral therapy and on pre-exposure prophylaxis for HIV. September 2015 Contract No.: NLM classification: WC 503.2.

Young, I., \& McDaid, L. (2014). How acceptable are antiretrovirals for the prevention of sexually transmitted HIV?: A review of research on the acceptability of oral pre-exposure prophylaxis and treatment as prevention. AIDS Behavior, $18,195-216$. 